\title{
AN IMPROVED BASELINE ESTIMATION METHOD USING EXTERNAL DEMS IN DIFFERENT TERRAIN AREAS
}

\author{
L. Feng, ${ }^{(1)}$ J.-P. Muller, ${ }^{(1)}$ Y.Li, ${ }^{(2)}$ M.Li $^{(4)}$ \\ ${ }^{(1)}$ Imaging Group, Mullard Space Science Laboratory (MSSL), University College London, Department of Space \& \\ Climate Physics, Holmbury St Mary, Surrey, RH5 6NT,UK, Email:lang.feng.14@ucl.ac.uk; j.muller@ucl.ac.uk \\ ${ }^{(2)}$ Key Laboratory for Aerial Remote Sensing Technology of National Administration of Surveying, Mapping and \\ Geoinformation(NASG) \& Chinese Academy of Surveying and Mapping(CASM), Email: \\ John_yuanwang_lee@hotmail.com \\ ${ }^{(3)}$ National Geomatics Centre of China , Email: liming210588@163.com
}

\begin{abstract}
Baseline estimation is a key parameter to calculate phase. The accuracy of baseline estimation has a direct impact on the final relative and absolute height accuracy in InSAR processing. This paper presents an improved baseline estimation method using an external DEM based FFT, which can be used in different terrain areas and deformation areas to obtain a relative good baseline estimation result.
\end{abstract}

\subsection{Introduction}

Space-borne Interferometric Synthetic Aperture Radar (InSAR) is being used as one of the innovative techniques for very accurate digital elevation model (DEM) generation. Interferometric phase, which includes ground phase, terrain phase, deformation phase, atmosphere phase and other phases, is the key to InSAR data processing. Moreover, in the processing, baseline estimation is a key parameter to calculate the ground phase, because the accuracy of the baseline estimation has a direct impact on the final relative and absolute height accuracy in InSAR processing. Normally, to obtain the DEM accuracy of InSAR at the metre level, the baseline estimate accuracy is required to achieve at least centimetre level.

Baseline estimation methods [1][2] can be grouped into the following categories: 1) baseline estimation using the Doppler equation, the distance equation and the earth ellipsoid equation; 2) the use of a precise orbit or registration rule; 3 ) using ground control points or a DEM; 4) assessment of the frequency of interference fringes in the ground plane. Previous studies have shown that baseline estimation based on the frequency of interference fringes requires the least information, but the accuracy is also the lowest. Baseline estimation using a precise orbit is completely dependent on the orbit, but a precise orbit is often difficult to obtain. Baseline estimation methods using Control points require a very large number of control points to guarantee a high enough baseline estimation accuracy. However, similar to the control points method, a DEM is probably the best choice to obtain the higher baseline estimation accuracy, because many global DEMs are now being produced and available from spaceborne EO sensors, such as from SAR (SRTM, TerraSAR-X, TanDEM-X), stereo-photogrammetric (ASTER, SPOT, PRISM and IRS-3P) and lidar (ICESat). This paper presents an improved external DEM based FFT baseline estimation method.

\section{Principles}

The typical geometry of InSAR taken from [2] is shown in the figure 1 below.
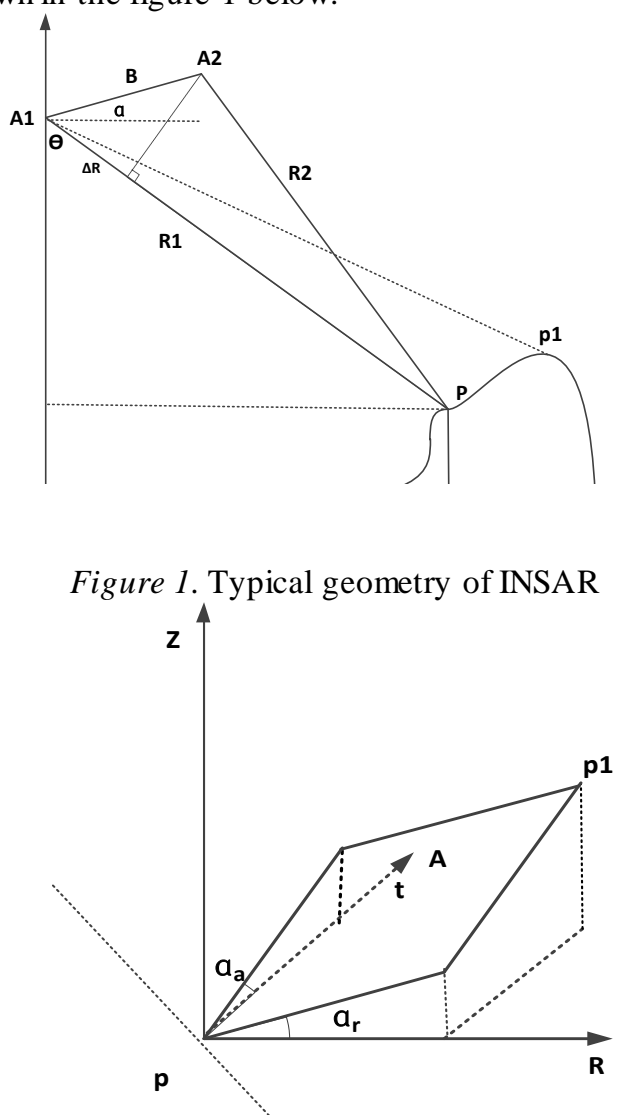

Figure 2. Two points p, p1 on ground in the radar coordinate system

As shown in Figure 1 above, A1 represents the first satellite image acquisition antenna position, A2 is the second satellite antenna position, the distance between $\mathrm{A} 1$ and $\mathrm{A} 2$ is the baseline, $\alpha$ represents the 
horizontal baseline angle, $\mathrm{H}$ represents the radar satellite's fly ing height, $\theta$ represents the line of sight viewing angle, $R 1$ represents the distance between $P$ and $\mathrm{A} 1, \mathrm{R} 2$ represents the distance between $\mathrm{P}$ and $\mathrm{A} 2$, $\Delta \mathrm{R}$ represents slant difference between $\mathrm{R} 2$ and $\mathrm{R} 1, \mathrm{~h}$ repres ents P's elevation.

According to the FFT method [3][6], a certain slant range $\left[r_{\min }, r_{\max }\right]$ is selected to perform the Fourier transform of the interferogram, the fringe frequency range is $\left[\mathrm{k}_{\min }, \mathrm{k}_{\max }\right]$. These equations are used to get baseline parameters $\mathrm{B}$ and $\mathrm{a}$. The equations of the FFT frequency method of interference fringes are shown in (1) - (3) below:

$$
\begin{aligned}
& k=-\frac{d \Phi}{d r} \\
& \mathrm{Bx}=\mathrm{B} \cdot \cos \alpha \\
& \mathrm{By}=\mathrm{B} \cdot \sin \alpha \\
& f(r)=\frac{4 \pi h}{\lambda r^{2}} \\
& g(r)=\frac{h}{\sqrt{r^{2}-h^{2}}} \\
& \left(\begin{array}{ll}
f\left(r_{\text {min }}\right) \cdot g\left(r_{\text {min }}\right) & f\left(r_{\text {min }}\right) \\
f\left(r_{\text {max }}\right) \cdot g\left(r_{\text {max }}\right) & f\left(r_{\text {max }}\right)
\end{array}\right)\left(\begin{array}{l}
B_{X} \\
B_{y}
\end{array}\right)=\left(\begin{array}{l}
\mathrm{k}_{\min } \\
\mathrm{k}_{\max }
\end{array}\right)
\end{aligned}
$$

Although the FFT method is very fast and has high accuracy, it should normally be applied in a flat area.

\section{Improved Method}

In order to study the phase relationship between any two points on the ground, the co-ordinate system shown in Figure 2 is used. SAR image coordinate system in [4] includes "quick time" in the $\tau$ axis direction, "slow time" for the $\mathrm{t}$-axis direction, assuming that the antenna along the $\mathrm{t}$ direction is the flight direction. Moreover, in the space Cartesian coordinate system, considering two points $\mathrm{P}(0,0,0)$ and $\mathrm{P} 1(\mathrm{a}, \mathrm{r}, \mathrm{z})$, $\alpha \mathrm{a}$ is the azimuth angle of slope, $\alpha \mathrm{r}$ is the range slope of slope. The complex interferogram frequency vector [4],[5] can be represented like this in equation (5), and Interferometric phase with the range and azimuth frequency is shown in (6) and (7) separately.

$$
\begin{aligned}
& \Delta f=\left[\begin{array}{ll}
\Delta f a & \Delta f r
\end{array}\right]=\frac{1}{2 \pi}\left[\begin{array}{ll}
\frac{\partial \phi}{\partial a} & \frac{\partial \phi}{\partial r}
\end{array}\right] \\
& \Delta f_{r}=\frac{1}{2 \pi} \frac{\partial \Delta \phi[t, \tau]}{\partial \tau}=-\frac{4 \pi}{\lambda} \frac{c}{2} \frac{B_{\perp}}{\tan \left(\theta-\alpha_{r}\right) R_{0}}
\end{aligned}
$$

$$
\Delta f_{a}=\frac{1}{2 \pi} \frac{\partial \Delta \phi[t, \tau]}{\partial t}=-\frac{2 f_{0} B_{\perp} V_{s}}{c R_{0}} \frac{\tan \alpha_{a} \tan \alpha_{r}}{\sin \left(\theta-\alpha_{r}\right)}
$$

Because the parallel baseline can be obtained directly from the SAR image distance to offset registration [5][6], the following discussion focuses on the estimated perpendicular baseline. To the range frequency, when $\alpha \mathrm{r}$ is 0 , Equation (6) can be changed to a new flat range fringe frequency shown in equation (8). It is then straightforward to obtain the perpendicular baseline shown in formula (9)

$$
\begin{aligned}
& \Delta f_{r-f l a t}=-\frac{4 \pi}{\lambda} \frac{c}{2} \frac{B_{\perp}}{\tan (\theta) R_{0}} \\
& B_{\perp}=-\frac{\Delta f_{r-f l a t} \lambda \tan (\theta) R_{0}}{2 \pi c}
\end{aligned}
$$

When the ground is pointing up or down, phase $\Delta \varphi(\mathrm{t}, \tau)$ contains two types of phase, flat phase and topographic phase, thus $\Delta \mathrm{fr}$ includes flat frequency $\Delta$ fr-flat and terrain frequency $\Delta$ fr-topo.

If the terrain phase can be removed from the total phase, flat phase is all that remains. Then the FFT algorith $m$ can be used to get an accurate baseline. More importantly, the residual phase by using a DEM, like the flat phase (after removing the flat phase and terrain phase) can also be used to get the residual baseline by using the FFT algorithm. Based on this idea, an improved DEM based FFT baseline estimation method shown in Fig. 3 is created. Firstly, the in itial baseline and interfero metric phase diagram by using master and slave image will use the initial baseline as the first iteration. Then, by subtracting the ideal phase diagram (generated from a coarse DEM simulation and the initial baseline with the track error) from the first interferometric phase diagram, residual errors will be extracted. In addition, the residual baseline is estimated from the residual fringe using the spatial frequency (FFT and Least squares method) method, and this is added to the initial baseline to do a further iteration to obtain a more accurate estimate of the baseline. If the residual phase includes extraordinary deformation phase, imaging analysis, like gradient ,etc., in range pixel line is used to avoid using the FFT algorith $m$ in these deformed areas and the Least squares method is also used with the FFT method in the range pixel line to get a more accurate residual baseline.

Slave image

4. Experimental results and analysis

The test data used are two Envisat ASAR ascending scenes (16/11/2003 and 25/01/2004), 
External DEMs used include SRTM3 and ASTER GDEM v2 and precise orbit data from ESA over Bam, Iran. We assume that the numbers in ESA's precise orbit are the true value for the track. After many iterations of the method, the perpendicular baseline error is $0.0457 \mathrm{~m}$ and the parallel baseline error is $0.0550 \mathrm{~m}$, which is better than the traditional Method.

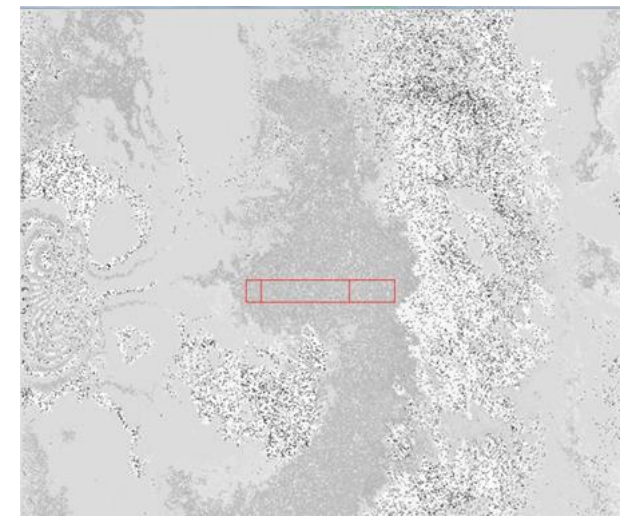

Figure 4. Range pixels for Least square and FFT in the second residual phase

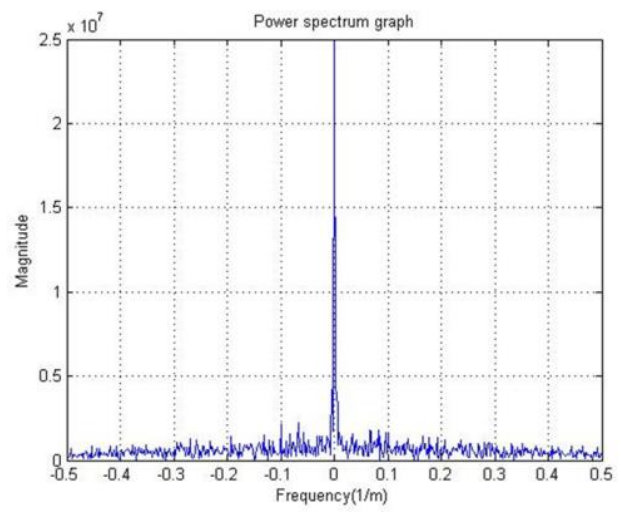

Figure 5. Phase power spectrum of the rectangle in fig 4

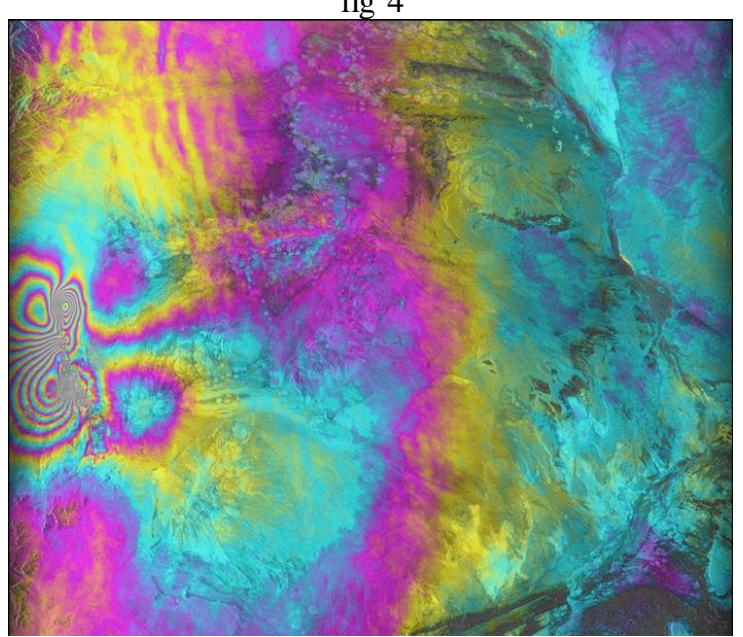

Figure 6. Interferogram of the method

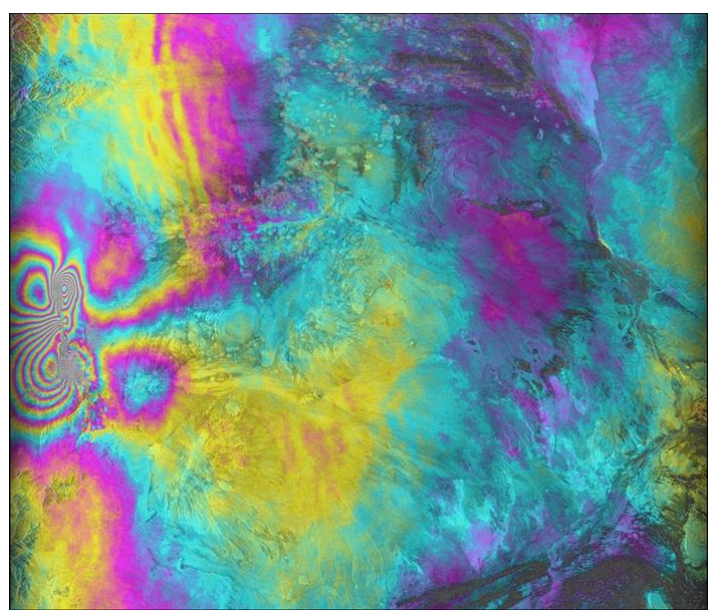

Figure 7. Interferogram of precise orbit

In our method, to avoid using the deformation phase in the FFT calculation, the range pixels (red rectangle shown in Fig4) need be selected wisely by any automated image analysis method. Fig 5 shows how to get the frequency of the phase interferogram of the rectangle area in Fig 4. Comparing the method's interferogram in Fig. 6 with the precise orbit method's interferogram in Fig 7, the method is more or less the same as the precise orbit method, which indicates that the method appears applicable to InSAR processing.

\section{Conclusions \& future work}

It is demonstrated that the baseline estimation accuracy can be improved by using an external DEM in some complex terrain areas. However, this method also has some shortcomings, like the phase caused by atmosphere, which requires to be solved in the future. In addition, external DEMs (TanDEM-X) with different resolution should be used, compared and assessed for baseline estimation to determine which DEM can be used in InSAR data processing in different areas.

\section{Acknowledgements}

China Scholarship Council (CSC) and University College London (UCL) jointly sponsor the work.

\section{References}

1. Ren Kun, Prinet V, and Shi Xiangquan, et al.. Comparison of satellite baseline estimation methods for interferometry appliacations[C]. Geoscience and Remote Sensing Symposium, 2003. IGA RSS 2003, Toulouse, France, 21-25 July 2003, Vol.6: 3821-3823.

2. $\mathrm{R}$ Bamler, $\mathrm{P}$ Hartl. Synthetic aperture radar interfero metry $[\mathrm{J}]$. Inverse Problems, 1998, 14(4) : R1- R54.

3. Singh K, Stussi N, Keong K L, et al. Baseline Estimation in Interferometric SAR[ C] IGARSS. 1997, 1997: 454- 456. 
4. Kohlhase A O, Feigl K L, Massonnet D. Applying Differential InSAR to Orbital Dynamics: A New Approach for Estimating ERS Trajectories[J]. Journal of Geodesy , 2003, 77:493-502.

5. WEI Hai-jun, ZHU Ju-bo, LIANG Dian-nong An Accurate Baseline Estimate Method for Rugged Terrain Utilize Rough DEM[J] Journal of National University of Defense Technology , 2010, 32(1) : 74- 78 .

6. Li X W, Guo H D, et al. Baseline estimation of interferometric SAR based on fast fourier transform[ J]. Acta Geodaetica et Cartographica Sinica, 2003, 32(1) : 70- 72. 